\title{
microRNA-135b expression silences Ppm1e to provoke AMPK activation and inhibit osteoblastoma cell proliferation
}

\author{
Zheng-Wei Li ${ }^{1, *}$, Yun-Rong Zhu ${ }^{2,}{ }^{,}$, Xiao-Zhong Zhou ${ }^{3,4, *}$, Bao-Biao Zhuo ${ }^{1}$, Xiao-Dong \\ Wang $^{1}$ \\ ${ }^{1}$ The Center of Diagnosis and Treatment for Children's Bone Diseases, The Children's Hospital Affiliated to Soochow University, \\ Suzhou, China \\ ${ }^{2}$ Department of Orthopedics, The Affiliated Jiangyin Hospital of Medical College of Southeast University, Jiangyin City, China \\ ${ }^{3}$ Department of Orthopedics, The Second Affiliated Hospital of Soochow University, Suzhou, China \\ ${ }^{4}$ Department of Orthopedics, The First People's Hospital of SuQian, SuQian, China \\ * Co-first authors
}

Correspondence to: Xiao-Dong Wang, email: xiaodongwangsz@163.com

Keywords: microRNA-135b, Ppm1e, AMPK, osteoblastoma, cell proliferation

Received: January 20, $2017 \quad$ Accepted: February 08, $2017 \quad$ Published: February 18, 2017

Copyright: Li et al. This is an open-access article distributed under the terms of the Creative Commons Attribution License (CC-BY), which permits unrestricted use, distribution, and reproduction in any medium, provided the original author and source are credited.

\section{ABSTRACT}

Forced-activation of AMP-activated protein kinase (AMPK) can possibly inhibit osteoblastoma cells. Here, we aim to provoke AMPK activation via microRNA silencing its phosphatase Ppm1e (protein phosphatase $\mathrm{Mg}^{2+} / \mathrm{Mn}^{2+}$-dependent 1e). We showed that microRNA-135b-5p ("miR-135b-5p"), the anti-Ppm1e microRNA, was significantly downregulated in human osteoblastoma tissues. It was correlated with Ppm1e upregulation and AMPKa1 de-phosphorylation. Forced-expression of miR-135b-5p in human osteoblastoma cells (MG-63 and U20S lines) silenced Ppm1e, and induced a profound AMPKa1 phosphorylation (at Thr-172). Osteoblastoma cell proliferation was inhibited after miR-135b-5p expression. Intriguingly, Ppm1e shRNA knockdown similarly induced AMPKa1 phosphorylation, causing osteoblastoma cell proliferation. Reversely, AMPKa1 shRNA knockdown or dominant negative mutation almost abolished miR-135b$5 p$ 's actions in osteoblastoma cells. Further in vivo studies demonstrated that U2OS tumor growth in mice was dramatically inhibited after expressing miR-135b-5p or Ppm1e shRNA. Together, our results suggest that miR-135b-induced Ppm1e silence induces AMPK activation to inhibit osteoblastoma cell proliferation.

\section{INTRODUCTION}

Osteoblastoma is one of the major causes of cancerrelated mortalities among children and young teenagers around the World [1-4]. Remarkable improvements have been achieved in the diagnosis and clinical treatment for osteoblastoma, and the five-year overall survival has been increased to over $70 \%$ [1-4]. However, for those with advanced, malignant and/or recurrent osteoblastoma, the prognosis is still very poor [1-4]. Therefore, our group has been dedicated to understanding the pathologic mechanisms of osteoblastoma tumorigenesis and progression, and developing novel anti-osteoblastoma agents $[5,6]$.

Recent studies have proposed a pivotal function of AMP-activate protein kinase (AMPK), the key energy sensor kinase [7], in regulating cell survival and death. It has been shown that AMPK activation could also inhibit cancer cells via regulating its downstream targeting proteins [8-12]. AMPK $\alpha 1$ subunit Thr-172 phosphorylation is vital for AMPK activation [7, 13, 14]. The underlying mechanism of phosphorylation of this site has been extensively studied [15]. Several AMPK upstream kinases, including LKB1 [14], CaMKK [16] and TAK1 [17], have been identified thus far. Yet, the phosphatase de-phosphorylates AMPK $\alpha 1$ is largely unknown. Recent studies have proposed that protein phosphatase $\mathrm{Mg}^{2+} / \mathrm{Mn}^{2+}$-dependent (Ppm) 1e (Ppm1e) could possibly a key AMPK $\alpha 1$ phosphatase [18-20]. Ppmle silence, inhibition and mutation were shown to induce AMPK activation via provoking AMPK $\alpha 1$ phosphorylation at Thr-172 [18-20]. 
microRNAs (miRNAs) are a group of endogenous small non-coding regulatory RNAs, which could inhibit gene expression at both translational and posttranscriptional levels $[21,22]$. miRNAs bind to the 3 ' untranslated region (UTR) of the targeted genes, causing mRNA decay as well as translational inhibition [21, 22]. Recent studies have identified a Ppmle-targeting miRNA: miR-135b-5p $[19,20]$. In the current study, we showed that miR-135b-5p expression silences Ppmle, which activates AMPK to inhibit osteoblastoma cell proliferation.

\section{RESULTS}

\section{miR-135b-5p upregulation correlates with Ppm1e upregulation and AMPKo1 de- phosphorylation in human osteoblastoma tissues}

First, we tested expression of miR-135b-5p, the Ppm1e-targeting miRNA $[19,20]$, in human osteoblastoma tissues. As demonstrated, miR-135b-5p expression level was significantly downregulated in osteoblastoma tissues ("Tumor", $n=10$ ), as compared to that in the surrounding normal bone tissues ("Normal", $n=10$ ) (Figure 1A). On the other hand, miR-135b-5p's target, Ppm1e mRNA, was upregulated in osteoblastoma tissues (Figure 1B). Ppm1e protein expression was also higher in osteoblastoma tissues than that in normal bone tissues (blot data were summarized in Figure 1C). As discussed above, Ppm1e is an established AMPK $\alpha 1$ phosphatase [18-20]. Thus, Ppmle upregulation shall cause AMPK $\alpha 1$ dephosphorylation. Indeed, the level of p-AMPK $\alpha 1$ (Thr-172) in the osteoblastoma tissues was much lower than that in the normal bone tissues (summarized in Figure 1D).

\section{Forced-expression of miR-135b-5p silences Ppm1e, causing AMPK activation in human osteoblastoma cells}

Next, a miR-135b expression vector ("Vec-miR$135 b$ ", a gift from Dr. Cui $[19,20])$ was introduced to MG-63 osteoblastoma cells. Via selection by puromycin, a total of three stable MG-63 cell lines ("L1/L2/L3") expressing Vec-miR-135b were established. qRT-PCR results in Figure 2A demonstrated that expression level of miR-135b-5p was indeed significantly upregulated in the stable cells ("L1/L2/L3"). On the other hand, miR-135b-3p level in above cells was quite low (Data not shown). Consequently, the Ppmle mRNA was depleted in the three lines (Figure 2B). Protein expression of Ppm1e in these cells was similarly downregulated (Figure 2C), causing AMPK activation or p-AMPK $\alpha 1$ increase (Figure $2 \mathrm{C}$ ). We also repeated the above experiments in another osteoblastoma cell line, U2OS cells. Once again, forcedexpression of miR-135b-5p in three U2OS cell lines ("L1/ L2/L3") (Figure 2D) led to Ppm1e depletion (Figure 2E and $2 \mathrm{~F}$ ) and significant AMPK activation (Figure 2F).
Introduction of the non-sense microRNA control ("mi-C") showed no effect on expressions of miR-135b, Ppm1e and p-AMPK $\alpha 1$ (Figure 2A-2F). Collectively, miR-135b-5p expression causes Ppm1e silence and AMPK activation in human osteoblastoma cells.

\section{Forced-expression of miR-135b-5p inhibits osteoblastoma cell proliferation}

Above results confirmed significant AMPK activation in miR-135b-expressed cells. As discussed above, activation of AMPK could possibly inhibit proliferation of many human cancer cells [23-26]. We thus tested the proliferation of the above osteoblastoma cells. Cell Counting Kit-8 (CCK-8) cell proliferation assay results in Figure 3A clearly showed that proliferation of MG-63 cells with miR-135b vector (three lines, "L1/L2/L3", see Figure 2) was significantly inhibited, as compared to cells with non-sense microRNA control ("mi-C") or empty vector (Figure 3A). Further, forced-expression of miR-135b-5p in MG-63 cells also dramatically decreased the number of colonies (Figure 3B). BrdU ELISA OD in these miR-135b$5 \mathrm{p}$-expressing cells was also decreased (Figure 3C). Very similar results were also obtained in U2OS cells. In the three lines ("L1/L2/L3") of miR-135b-5p-expressing U2OS cells, CCK-8 OD (Figure 3D), colony formation (Figure 3E) and BrdU incorporation ELISA OD (Figure 3F) were all decreased. Introduction of the non-sense microRNA control ("mi-C") had no such effect on the osteoblastoma cells (Figure 3A-3F). These results demonstrate that forced miR135b-5p expression inhibits osteoblastoma cell proliferation.

\section{Ppm1e shRNA knockdown activates AMPK and inhibits osteoblastoma cell proliferation}

Above results have shown that forced-expression of miR-135b-5p silenced Ppm1e and inhibited osteoblastoma cell proliferation. If Ppmle is the direct target of miR-135b-5p, knockdown of Ppm1e shall also inhibit osteoblastoma cell proliferation. To test this hypothesis, a panel of two distinct lentiviral Ppm1e shRNAs ("shPpm1e-1" and "shPpm1e-1", gifts from Dr. Cui's group [20], were employed. Results showed that the applied Ppm1e shRNAs efficiently silenced Ppm1e in MG63 cells (Figure 4A and 4B). As a result, AMPK activation, or p-AMPK $\alpha 1$, was increased (See quantified results in Figure 4B). Expression of miR-135b-5p, as expected, was unchanged in Ppm1e-silenced cells (Figure 4C). Remarkably, proliferation of MG-63 cells, tested again by the CCK-8 assay (Figure 4D) and colony formation assay (Figure 4E), was inhibited with Ppmle shRNA knockdown. The scramble shRNA control ("sh-C") didn't change Ppm1e expression, miR-135b-5p level, AMPK activation and MG-63 cell proliferation (Figure 4A-4E).

Based on the results above, we speculated that miR-135b-5p could possibly be invalid in Ppm1e- 
depleted cells. Thus, we therefore exogenously expressed miR-135b vector in the two MG-63 lines with Ppm1e shRNA ("shPpm1e-1" and "shPpm1e-1"). As shown in Figure 4F, the level of miR-135b-5p was again elevated after expression of vector. Yet, unlike control cells (See
Figure 3), forced-expression of miR-135b-5p failed to further inhibit the proliferation of Ppm1e-silneced cells (Figure 4G). These results together imply that Ppm1e is the direct and primary target of miR-135b-5p in mediating its actions in osteoblastoma cells.
A

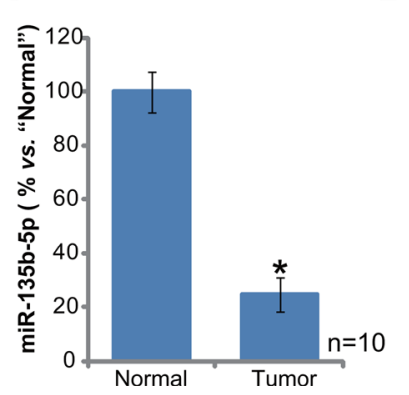

B

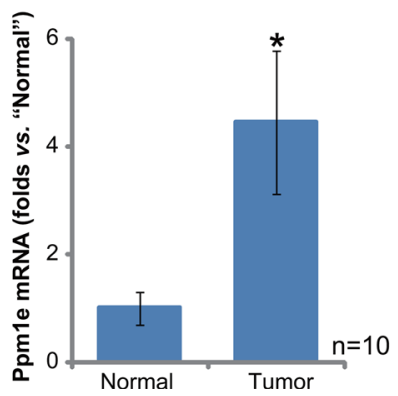

C

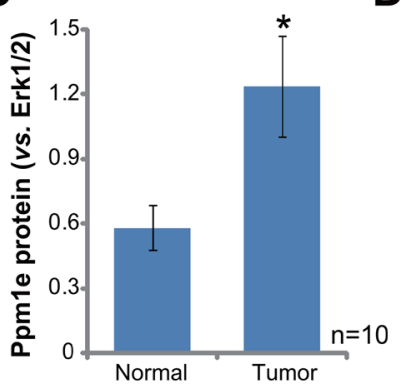

D

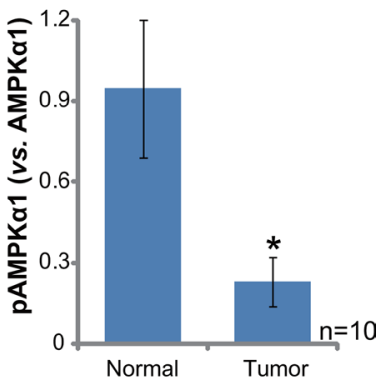

Figure 1: miR-135b-5p upregulation correlates with Ppm1e upregulation and AMPKa1 de-phosphorylation in human osteoblastoma tissues. Expressions of miR-135b-5p (A, qRT-PCR assay), Ppm1e mRNA (B, qRT-PCR assay) and listed proteins (C-D, Western blotting assay) in ten (10) different human osteoblastoma tissues ("Tumor") and surrounding normal bone tissues ("Normal") were tested. Ppm1e protein expression ( $v s$. Erk1/2) and AMPK $\alpha 1$ phosphorylation ( $v s$. total AMPK $\alpha 1)$ were quantified $(\mathbf{C}-\mathbf{D})$. ${ }^{*} p<0.05$ vs. "Normal" group.

A MG-63 B

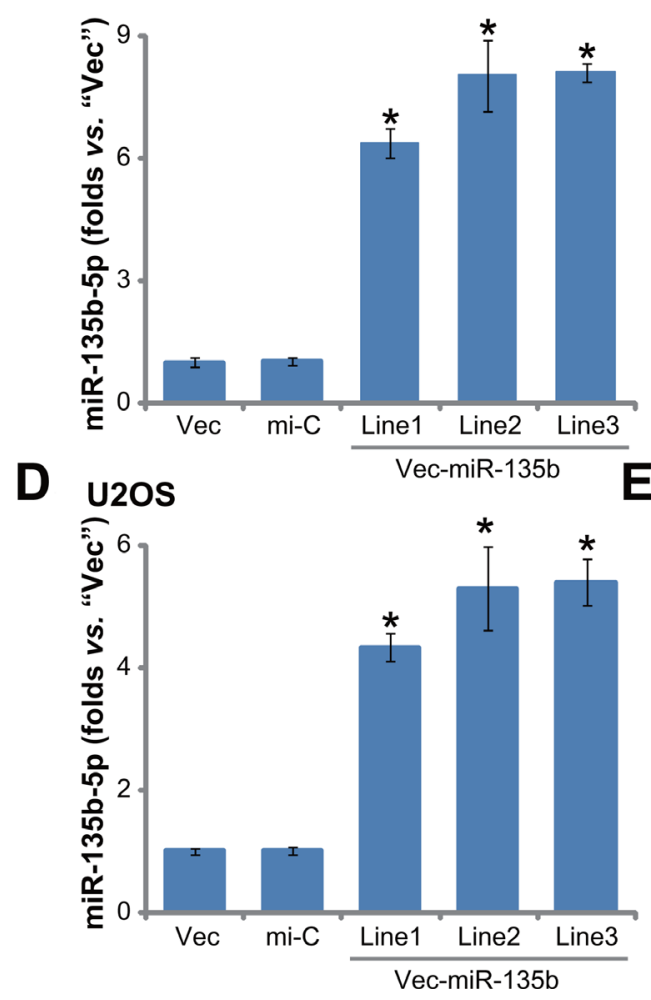

C
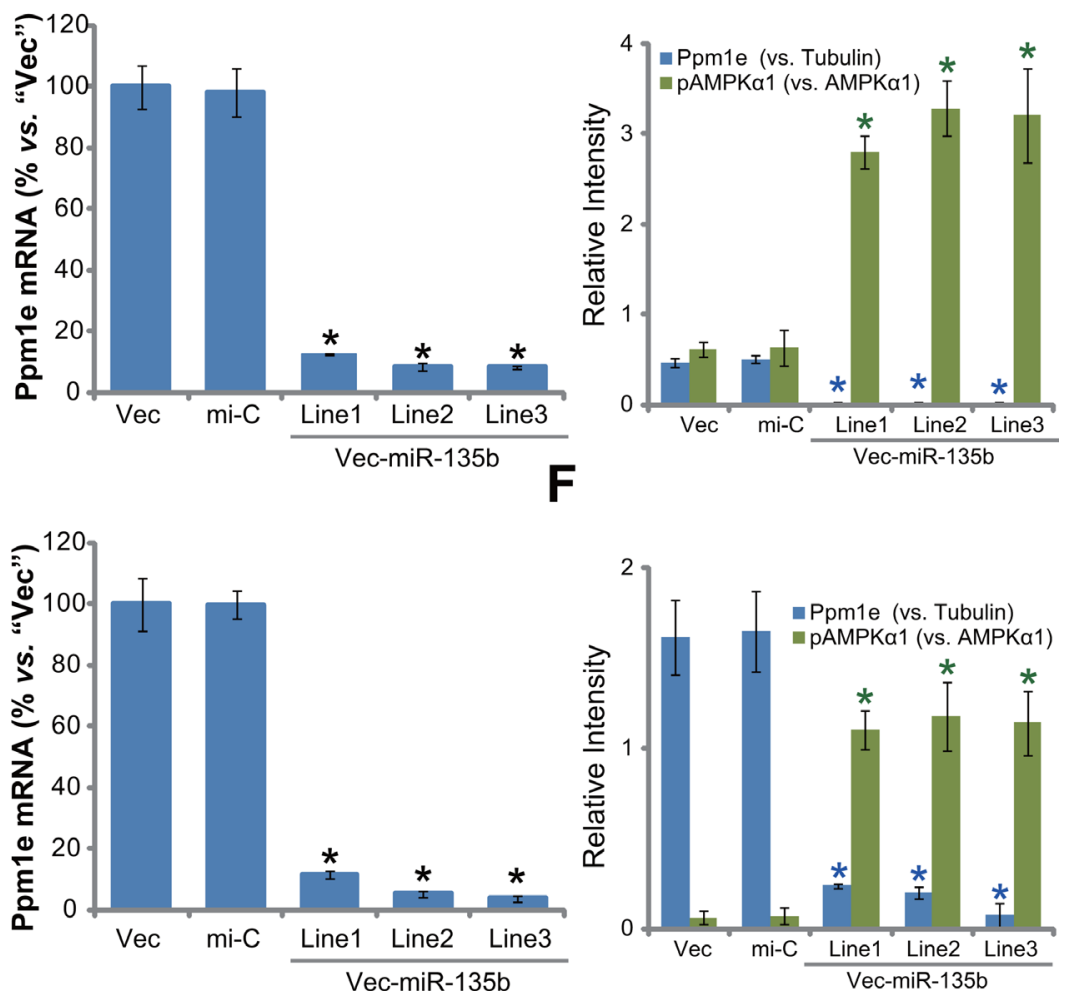

$\mathbf{F}$

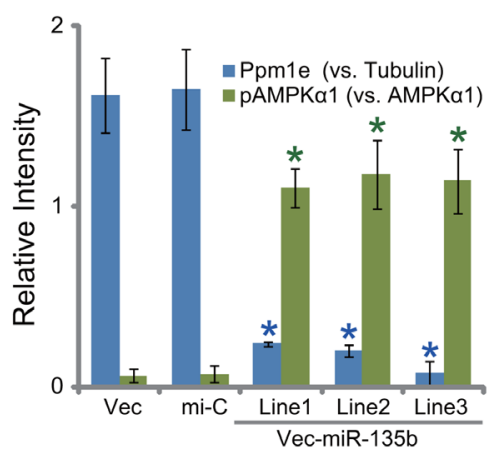

Figure 2: Forced-expression of miR-135b-5p silences Ppm1e, causing AMPK activation in human osteoblastoma cells. Stable MG-63 cells (A-C) or U2OS cells (D-F), expressing miR-135b vector ("Vec-miR-135b", three lines each, "L1/L2/L3"), microRNA control ("mi-C") vector or the empty vector ("Vec", pSuper-puro), were subjected to qRT-PCR assay to test expression of miR-135b-5p (A and D) and Ppmle mRNA (B and E); Listed proteins in above cells were tested by Western blot assay, Ppm1e protein expression ( $v s$. Tubulin) and AMPK $\alpha 1$ phosphorylation ( $v s$. total AMPK $\alpha 1$ ) were quantified (C and F). For each assay, $n=5$. * $p<0.05 v s$. group "mi-C". Experiments in this figure were repeated three times, and similar results were obtained. 


\section{AMPKa1 shRNA knockdown or mutation abolishes miR-135b-5p's actions against osteoblastoma cells}

Above results showed that forced-expression of miR-135b-5p activated AMPK signaling and inhibited osteoblastoma cell proliferation. We thus wanted to know the association between the two. In order to block AMPK activation, AMPK $\alpha 1$ shRNA [20, 27] or a dominant negative mutation of AMPK $\alpha 1$ (T172A, Flag-tagged) $[20,27]$ was introduced to vec-miR-135bexpressing MG-63 cells ("L1", see Figure 2). As shown in Figure 5A, AMPKa1 shRNA knockdown or T172A mutation almost completely blocked AMPK activation (p-AMPK $\alpha 1$ at Thr-172) in vec-miR-135b-expressing cells. Ppm1e expression (Figure 5A) and miR-135b-5p level (Figure 5B) were unchanged in AMPK $\alpha 1$-silenced or -mutant cells. Remarkably, miR-135b-5p-induced MG-63 cell proliferation inhibition was almost reversed by AMPK $\alpha 1$ knockdown or mutation (Figure 5C and 5D). These results suggest that activation of AMPK is required for miR-135b-5p-induced MG-63 cell proliferation inhibition. We repeated the experiments in U2OS cells, and similar results were obtained (Data not shown).

\section{U2OS tumor growth in SCID mice is inhibited after expressing Ppm1e shRNA or miR-135b-5p}

The potential effect of miR-135b-5p on osteoblastoma cell growth in vivo was tested. U2OS cells, stably expressing Ppmle shRNA (“-1", see Figure 4), VecmiR-135b ("L1", see Figure 2) or empty vector ("Vec", see Figure 2), were inoculated into the severe combined immunodeficient (SCID) mice via s.c. injection. Tumor recordings were started when the tumors were around $100 \mathrm{~mm}^{3}$ in volume for each group. Tumor growth curve results in Figure 6A demonstrated that growth of U2OS tumors was largely inhibited after expressing Ppm1e shRNA or Vec-miR-135b. Estimated daily tumor growth (in $\mathrm{mm}^{3}$ per day) was also significantly lower in Ppm1e shRNA- or Vec-miR-135b-expressing tumors (Figure 6B). At the end of the experiments (Week-7), the weight of tumors with Ppm1e shRNA or Vec-miR-135b was also much lower than "Vec" control tumors (Figure 6C). The mice body weight was indifferent between the three groups (Figure 6D). At Week-7, all xenografted tumors were isolated and lysed. Expressions of miR-135b-5p and Ppm1e were examined. As demonstrated, Ppm1e mRNA was indeed depleted in Ppmle shRNA- or Vec-
A $M G-63$

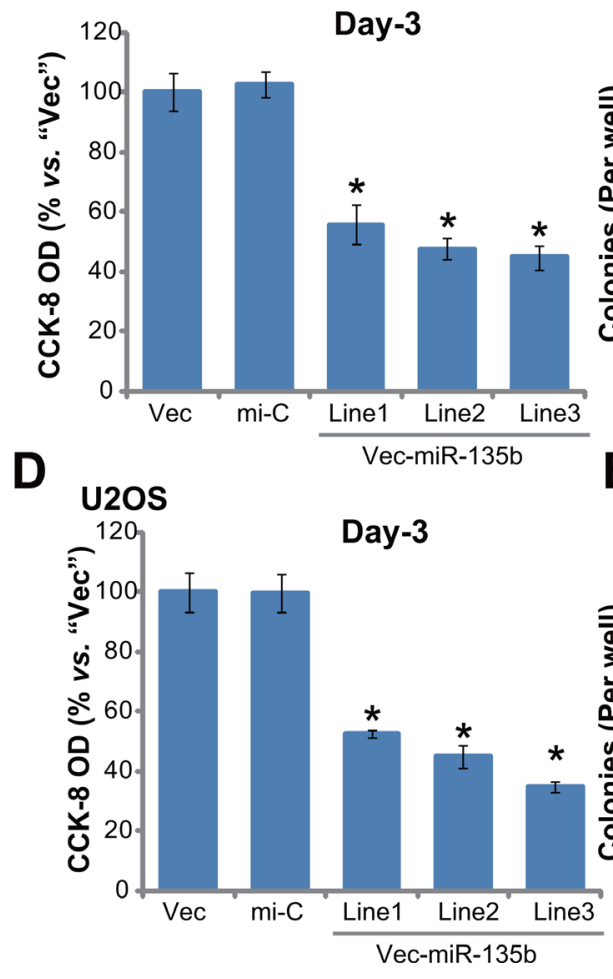

B
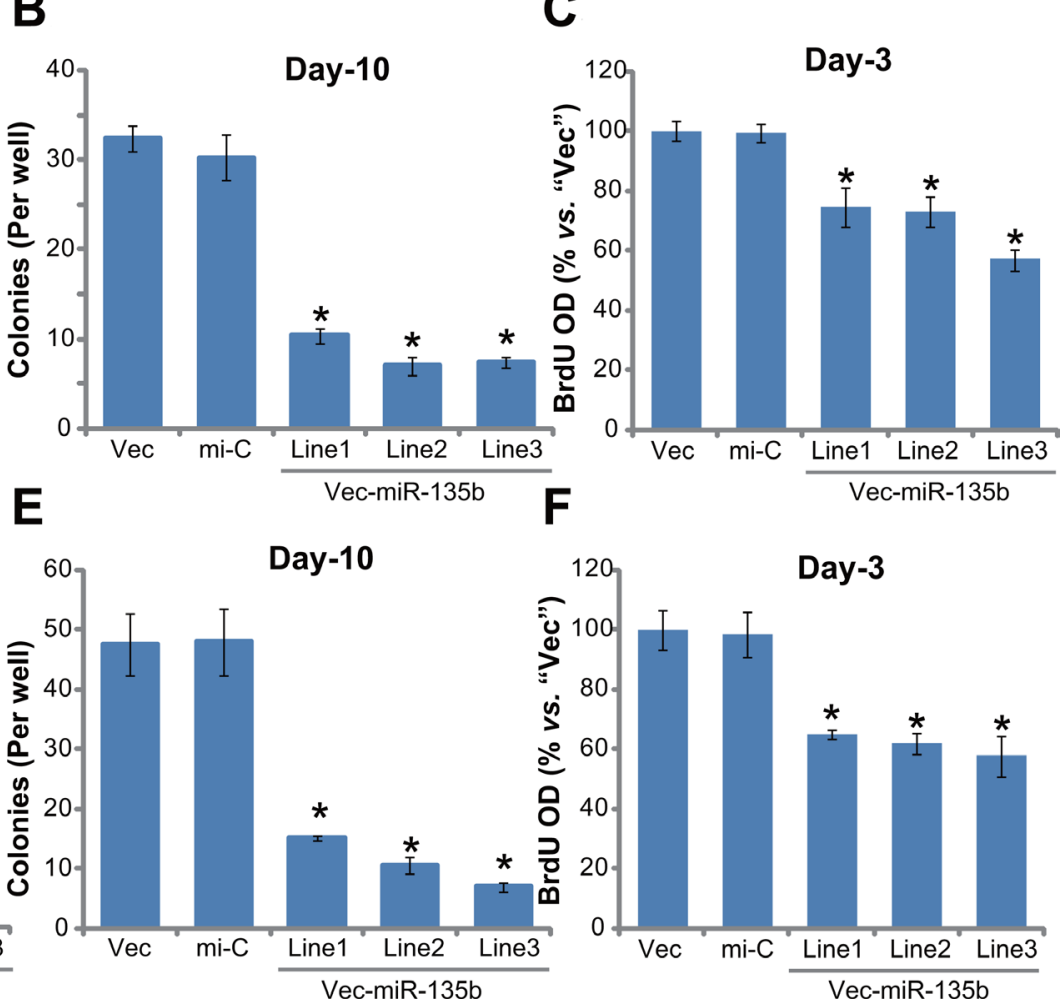

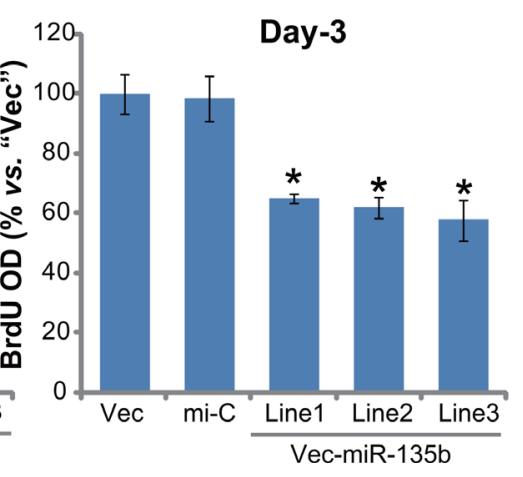

Figure 3: Forced-expression of miR-135b-5p inhibits osteoblastoma cell proliferation. Stable MG-63 cells (A-C) or U2OS cells (D-F), expressing miR-135b vector ("Vec-miR-135b", three lines each, "L1/L2/L3"), microRNA control ("mi-C") vector or the empty vector ("Vec", pSuper-puro), were subjected to following cell proliferation assays: CCK-8 assay (A and D), colony formation assay (B and E) and BrdU incorporation assay ( $\mathrm{C}$ and $\mathrm{F}$ ). Notably, exact same number of viable cells of different background were plated initially for these proliferation assays (Same for all Figures). For each assay, $n=5$. ${ }^{*} p<0.05$ vs. group "mi-C". Experiments in this figure were repeated three times, and similar results were obtained. 
miR-135b-expressing tumors (Figure 6E). miR-135b-5p was upregulated in Vec-miR-135b-expressing tumors (Figure 6F). Together, these results indicate that U2OS tumor growth in SCID mice is inhibited after expressing Ppmle shRNA or miR-135b-5p.

\section{DISCUSSION}

Here, we demonstrated that miR-135b-5p expression was downregulated in human osteoblastoma tissues, which was associated with Ppmle upregulation. Forced-expression of miR-135b-5p silenced Ppm1e and potently inhibited osteoblastoma cell proliferation in vitro and in vivo. These results imply that miR-135b-5p could possibly be a novel anti-cancer microRNA ("antioncomir") in osteoblastoma. Remarkably, we imply that AMPK activation could be responsible for miR-135b5p-mediated anti-osteoblastoma cell activity. In cultured human osteoblastoma cells (MG-63 and U2OS lines), forced-expression of miR-135b depleted Ppm1e, leading

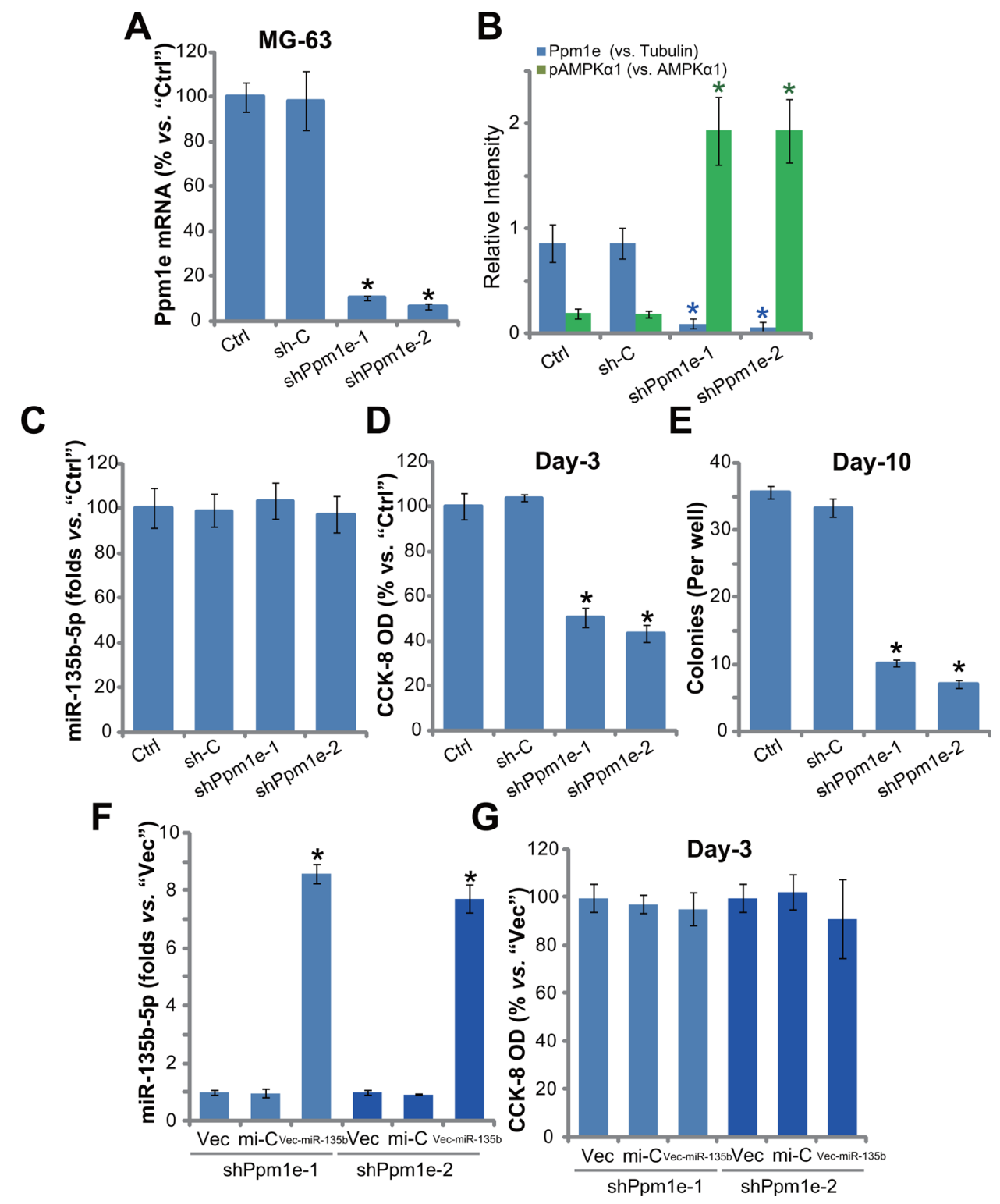

Figure 4: Ppm1e shRNA knockdown activates AMPK and inhibits osteoblastoma cell proliferation. Stable MG-63 cells with listed Ppm1e shRNA ("shPpm1e-1" or "shPpm1e-1") or scramble shRNA control ("sh-C"), as well as the parental control MG-63 cells ("Ctrl") were subjected to qRT-PCR assay to test expression of Ppm1e mRNA (A) and miR-135b-5p (C); Listed proteins in above cells were also tested, and blot data were quantified (B). Proliferation of above cells was tested by the CCK-8 assay (D) and colony formation assay (E). MG-63 cells with listed Ppm1e shRNA ("shPpm1e-1" or "shPpm1e-1") were also transfected with miR-135b vector ("VecmiR-135b"), expression of miR-135b-5p was tested afterwards (F); Cell proliferation was examined by CCK-8 assay (G). Ppm1e protein expression (vs. Tubulin) and AMPK $\alpha 1$ phosphorylation (vs. total AMPK $\alpha 1$ ) were quantified (B). Notably, exact same number of viable cells of different background were plated initially for these proliferation assays. For each assay, $n=5$. * $p<0.05 v s$. group "sh-C"/“mi-C". Experiments in this figure were repeated three times, and similar results were obtained. 
to profound AMPK activation (AMPK $\alpha 1$ phosphorylation at Thr-172). Importantly, AMPK $\alpha 1$ shRNA knockdown or dominant negative mutation almost abolished miR-135b$5 \mathrm{p}$-induced actions in osteoblastoma cells.

Intriguingly, we here proposed that Ppmle is the primary target of miR-135b in mediating its inhibition against osteoblastoma cells. shRNA-mediated knockdown of Ppm1e mimicked miR-135b-5p's actions, and similarly induced AMPK activation and inhibited osteoblastoma cell proliferation. Remarkably, forced-expression of miR-135b-5p failed to further inhibit the proliferation of Ppm1e-silneced cells. Therefore, miR-135b-5p expression was in-effective when Ppmle was depleted, suggesting that Ppmle is the direct target of this Anti-

\section{A}

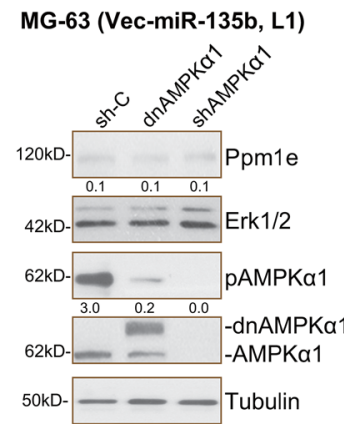

B

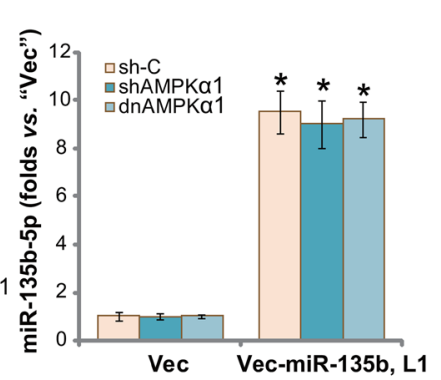

C

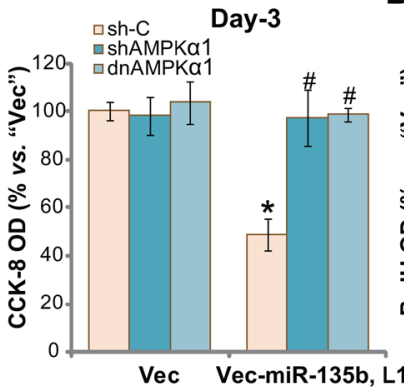

D

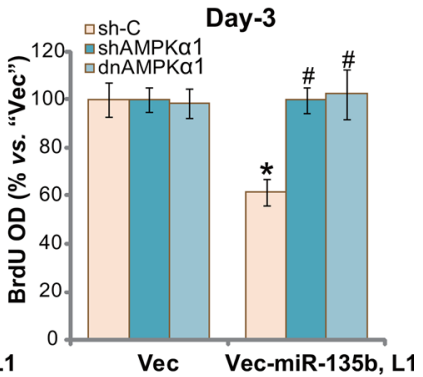

Figure 5: AMPKa1 shRNA knockdown or mutation abolishes miR-135b-5p's actions against osteoblastoma cells. Stable MG-63 cells with miR-135b vector ("Vec-miR-135b", "L1") or the empty vector ("Vec", pSuper-puro) were further constructed with AMPK $\alpha 1$ shRNA ("shAMPK $\alpha 1$ "), scramble control shRNA ("sh-C") or a dominant negative mutation of AMPK $\alpha 1$ (T172A, Flagtagged, ““dnAMPK $\alpha 1$ ”), expressions of listed proteins were shown (A); miR-135b-5p mRNA expression was tested by qRT-PCR assay (B); Cell proliferation was tested by the CCK-8 assay (C) and BrdU ELISA assay (D). Ppm1e protein expression ( $v s$. Erk1/2) and AMPK $\alpha 1$ phosphorylation (vs. Tubulin) were quantified (A). For each assay, $n=5$. ${ }^{*} p<0.05$ vs. group "Vec" only. " $p<0.05 v s$. group "sh-C". Experiments in this figure were repeated three times, and similar results were obtained.

A

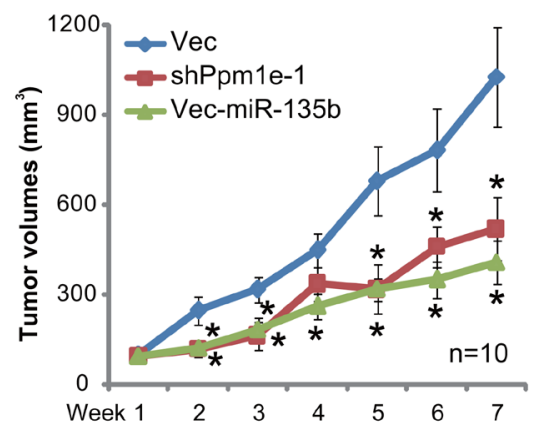

D

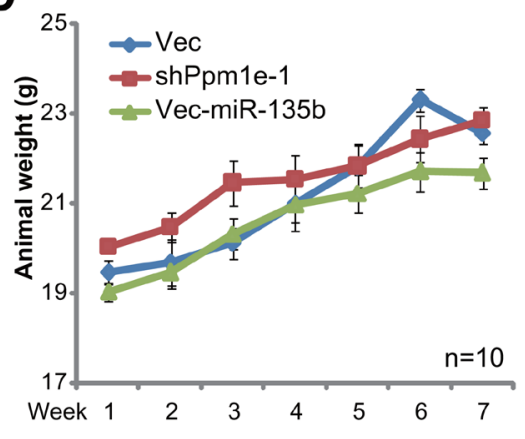

B

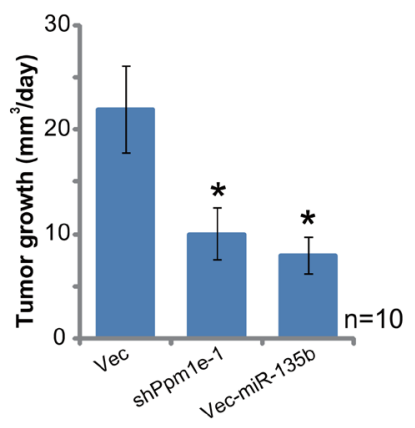

$\mathbf{E}$

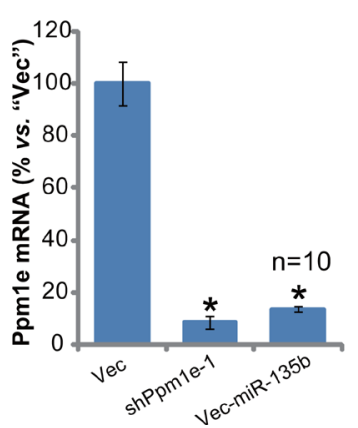

C

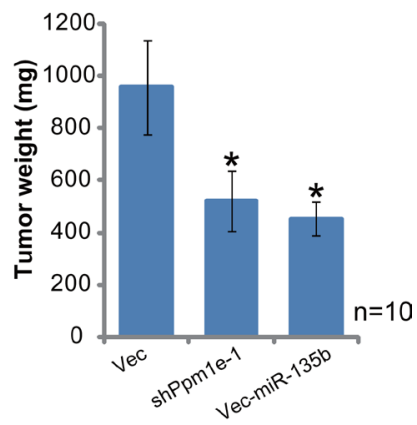

$\mathbf{F}$

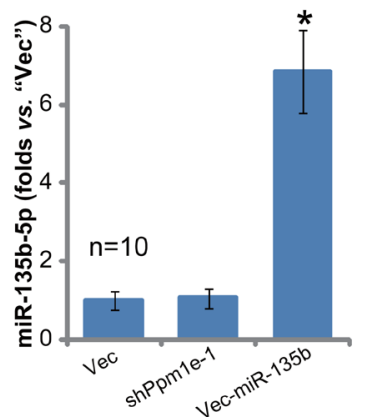

Figure 6: U2OS tumor growth in SCID mice is inhibited after expressing Ppm1e shRNA or miR-135b-5p. Stable U2OS cells, with Ppm1e shRNA ("-1"), Vec-miR-135b ("L1") or empty vector ("Vec"), were inoculated into the SCID mice via s.c. injection. When the tumors were around $100 \mathrm{~mm}^{3}$ in volume, the recording were started. Tumor volume (A) and mice body weight (D), which subtracting estimated tumor weight) were recorded every week for a total of 6 weeks (Week1 to Week7); Estimated daily tumor growth, in $\mathrm{mm} 3$ per day, was also calculated (B); At week-7, tumors of each group were isolated and weighted (C); Expressions of Ppm1e mRNA $(\mathbf{E})$, miR-135b-5 (F) in tumor tissue lysates were also tested. For each group, $n=10 .{ }^{*} p<0.05$ vs. "Vec" control tumors. 
oncomir. Notably, we showed that Ppmle expression was significantly elevated in osteoblastoma tissues. On the other hand, shRNA-mediated Ppm1e knockdown potently inhibited osteoblastoma cell growth in vivo and in vivo. These results indicate that Ppm1e could be a novel oncotarget protein of osteoblastoma.

It is well-known that AMPK participates in gene transcription, cell mitosis, apoptosis and autophagy $[13,28]$. Sustained and profound AMPK activation shall inhibit cell growth, and promote cell death and apoptosis [29]. The cancer-inhibitory activity by AMPK is achieved through regulating one or multiple AMPK downstream signal target proteins, including mTOR inhibition [30-33], p53/p27 activation [34-37], JNK activation [35, 38-40] and Ulk1-autophagy pathway [41]. Indeed, many cytotoxic chemo-agents, including vincristine [36, 42], taxol [39, 43], temozolomide [34], and doxorubicin $[30,35]$, all activate AMPK signaling to kill cancer cells. Therefore, further studies will be needed to explore the downstream signalings of AMPK that mediate miR-135b's actions in osteoblastoma cells.

\section{MATERIALS AND METHODS}

\section{Chemicals and reagents}

The Ppm1e antibody was provided by Dr. Cui's group at Nantong University [20]. All other antibodies were obtained from Cell Signaling Tech (Denver MA). The enhanced chemiluminescence (ECL) reagents were obtained from Pierce (Rockford, IL). The cell culture reagents were provided by Gibco Co. (Shanghai, China).

\section{Cell culture}

As described [44], osteoblastoma U2OS and MG-63 cells were cultured in DMEM plus FBS medium [6].

\section{Human tissue specimens}

As described previously $[5,6]$, the surgery-isolated osteoblastoma tissues and surrounding normal bone tissues were separated carefully. Tissues were washed thoroughly and minced into small pieces. Tissues were then mechanically dissociated and lysed by the descried tissue lysis buffer $[5,6]$. Tissue lysates were stored in liquid nitrogen. This study was conducted according to the principles expressed in the Declaration of Helsinki. The protocol was approved by Internal Review Board and Ethic Review Board of Soochow University. Writteninformed consent was obtained from each participant.

\section{CCK-8 cell proliferation assay}

Cell Counting Kit-8 (CCK-8) (Dojindo, Japan) assay was employed to test cell proliferation according to the attached instructions. The detailed protocol was described in our previous study [44].

\section{Other cell proliferation assays}

Clonogenicity assay of cell growth and BrdU ELISA assay of cell proliferation were described in detail in our previous studies $[44,45]$.

\section{Western blotting assay}

Cell and tissue lysates (40 $\mu \mathrm{g} /$ sample) were separated by $10-12 \%$ SDS-PAGE gel, and were transferred onto PVDF membrane (Millipore, USA). Afterwards, the membrane was blocked, followed by incubation with specific primary and corresponding secondary antibodies. The detection was performed via ECL Supersingnal West Pico Chemiluminescent. Indicated band was quantified via ImageJ software.

\section{Quantitative real-time PCR assay}

The protocol of quantitative real-time reverse transcriptase polymerase chain reaction ("qRT-PCR") assay was described in detail in our previous studies $[6,44,45]$. We utilized the comparative $\mathrm{Ct}\left(2^{-\Delta \Delta \mathrm{Ct}}\right)$ method to quantify target mRNA expression [46]. GAPDH was tested as the reference gene [6]. mRNA primers for GAPDH and Ppm1e were provided by Dr. Cui [20]. The expression of mature miR-135b was tested by the TaqMan microRNA assay as described [47]. Ten ng of total RNA per sample was reverse-transcribed via the TaqMan MicroRNA Reverse Transcription Kit (Applied Biosystem, Shanghai, China) [47]. The miR-135b-5p primers were described early [48, 49].

\section{Forced-expression of miR-135b}

The miR-135b pSuper-puro-GFP vector ("Vec-miR$135 \mathrm{~b}$ ") and miR-control ("miR-C") vector were provided by Dr. Cui [20]. Cells were transfected with the vector by Lipofectamine 2000 reagent (Invitrogen). After 24 hours, cells were subjected to puromycin $(1 \mu \mathrm{g} / \mathrm{mL})$ selection for another 72 hours. miR-135b-5p expression in the resulting stable cells was tested by qRT-PCR assay.

\section{shRNA knock and stable cell selection}

The two distinct lentiviral Ppmle shRNAs were provided again by Dr. Cui [20]. The scramble control shRNA (sc-108065) and AMPK $\alpha 1$ shRNA (sc-45312-V) were purchased from Santa Cruz Biotech (Shanghai, China). Osteoblastoma cells were seeded onto six-well plates with $50-60 \%$ confluence. Ten $\mu \mathrm{L} / \mathrm{mL}$ of lentiviral shRNA was added directly to cultured cells for 24 hours. Afterwards, puromycin ( $1 \mu \mathrm{g} / \mathrm{mL}$, Sigma) was added to the culture medium for another 48 hours. The knockdown 
of targeted protein (Ppmle or AMPK $\alpha 1$ ) was verified by Western blotting assay and/or qRT-PCR assay.

\section{AMPK dominant negative mutation}

The dominant negative mutant of AMPK $\alpha 1$ (dnAMPK $\alpha 1$, T172A, Flag-tagged) construct was provided by Dr. Lu [8]. Cells were seeded onto six-well plate with 50-60\% confluence. The dn-AMPK $\alpha 1$ vector was transfected to cells using Lipofectamine 2000 protocol [8], and stable cells were selected via puromycin $(1 \mu \mathrm{g} / \mathrm{mL}$, Sigma).

\section{Mice U2OS xenograft assay}

As described [5, 50, 51], CB.17 severe combined immuno-deficient (SCID) male mice (18-20 g) were purchased from the Animal Facility of Soochow University (Suzhou, China). Three million of U2OS cells (per mouse), with Ppm1e shRNA or "Vec-miR-135b", were inoculated subcutaneously (s.c.) into the flanks of the mice. When the xenografts were about $100 \mathrm{~mm}^{3}$ in volume, recordings were starte as described [50-52]. The protocols were in accordance with the Institutional Animal Care and Use Committee (IACUC), and were approved by the Ethics Committee and Internal Review Board (IRB) of Soochow University.

\section{Statistical analysis}

The quantitative data presented in this study was mean \pm standard deviation (SD). Statistical differences were analyzed by one-way ANOVA with post hoc Bonferroni test.

\section{CONCLUSIONS}

We conclude that miR-135b silences Ppm1e to provoke AMPK activation and inhibit osteoblastoma cell proliferation.

\section{ACKNOWLEDGMENTS AND FUNDING}

The study was supported by the Nature Science Foundation of China. Grants from Natural Science Foundation of Jiangsu Province, and Young medical talents of Jiangsu Province (QNRC2016132).

\section{CONFLICTS OF INTEREST}

The authors have no conflicts of interests.

\section{REFERENCES}

1. Isakoff MS, Bielack SS, Meltzer P, Gorlick R. Osteosarcoma: Current Treatment and a Collaborative Pathway to Success. J Clin Oncol. 2015; 33:3029-3035.

2. Kansara M, Teng MW, Smyth MJ, Thomas DM. Translational biology of osteosarcoma. Nat Rev Cancer. 2014; 14:722-735.
3. Yang J, Zhang W. New molecular insights into osteosarcoma targeted therapy. Curr Opin Oncol. 2013; 25:398-406.

4. Broadhead ML, Clark JC, Myers DE, Dass CR, Choong PF. The molecular pathogenesis of osteosarcoma: a review. Sarcoma. 2011; 2011:959248.

5. Zhen YF, Li ST, Zhu YR, Wang XD, Zhou XZ, Zhu LQ. Identification of DNA-PKcs as a primary resistance factor of salinomycin in osteosarcoma cells. Oncotarget. 2016; 7:79417-79427. doi: 10.18632/oncotarget.12712.

6. Zhu LQ, Zhen YF, Zhang Y, Guo ZX, Dai J, Wang XD. Salinomycin activates AMP-activated protein kinasedependent autophagy in cultured osteoblastoma cells: a negative regulator against cell apoptosis. PLoS One. 2013; 8:e84175.

7. Hardie DG, Ross FA, Hawley SA. AMPK: a nutrient and energy sensor that maintains energy homeostasis. Nat Rev Mol Cell Biol. 2012; 13:251-262.

8. Lu PH, Chen MB, Ji C, Li WT, Wei MX, Wu MH. Aqueous Oldenlandiadiffusa extracts inhibits colorectal cancer cells via activating AMP-activated protein kinase signalings. Oncotarget. 2016; 7:45889-45900. doi: 10.18632/ oncotarget.9969.

9. Chen MB, Zhang Y, Wei MX, Shen W, Wu XY, Yao C, $\mathrm{Lu} \mathrm{PH}$. Activation of AMP-activated protein kinase (AMPK) mediates plumbagin-induced apoptosis and growth inhibition in cultured human colon cancer cells. Cell Signal. 2013; 25:1993-2002.

10. Chen MB, Wei MX, Han JY, Wu XY, Li C, Wang J, Shen W, Lu PH. MicroRNA-451 regulates AMPK/mTORC1 signaling and fascin 1 expression in HT-29 colorectal cancer. Cell Signal. 2014; 26:102-109.

11. Chen MB, Shen WX, Yang Y, Wu XY, Gu JH, Lu PH. Activation of AMP-activated protein kinase is involved in vincristine-induced cell apoptosis in B16 melanoma cell. J Cell Physiol. 2010; 226:1915-1925.

12. Chen MB, Jiang Q, Liu YY, Zhang Y, He BS, Wei MX, Lu JW, Ji Y, Lu PH. C6 ceramide dramatically increases vincristine sensitivity both in vivo and in vitro, involving AMP-activated protein kinase-p53 signaling. Carcinogenesis. 2015; 36:1061-1070.

13. Mihaylova MM, Shaw RJ. The AMPK signalling pathway coordinates cell growth, autophagy and metabolism. Nat Cell Biol. 2011; 13:1016-1023.

14. Shaw RJ, Kosmatka M, Bardeesy N, Hurley RL, Witters LA, DePinho RA, Cantley LC. The tumor suppressor LKB1 kinase directly activates AMP-activated kinase and regulates apoptosis in response to energy stress. Proc Natl Acad Sci U S A. 2004; 101:3329-3335.

15. Hardie DG. AMP-activated/SNF1 protein kinases: conserved guardians of cellular energy. Nat Rev Mol Cell Biol. 2007; 8:774-785.

16. Jensen TE, Rose AJ, Jorgensen SB, Brandt N, Schjerling P, Wojtaszewski JF, Richter EA. Possible CaMKK-dependent regulation of AMPK phosphorylation and glucose uptake at 
the onset of mild tetanic skeletal muscle contraction. Am J Physiol Endocrinol Metab. 2007; 292:E1308-1317.

17. Herrero-Martin G, Hoyer-Hansen M, Garcia-Garcia C, Fumarola C, Farkas T, Lopez-Rivas A, Jaattela M. TAK1 activates AMPK-dependent cytoprotective autophagy in TRAIL-treated epithelial cells. EMBO J. 2009; 28:677-685.

18. Voss M, Paterson J, Kelsall IR, Martin-Granados C, Hastie CJ, Peggie MW, Cohen PT. Ppm1E is an in cellulo AMP-activated protein kinase phosphatase. Cell Signal. 2011; 23:114-124.

19. Li P, Fan JB, Gao Y, Zhang M, Zhang L, Yang N, Zhao X. miR-135b-5p inhibits LPS-induced TNFalpha production via silencing AMPK phosphatase Ppm1e. Oncotarget. 2016; 7:77978-77986. doi: 10.18632/oncotarget.12866.

20. Fan JB, Ruan JW, Liu W, Zhu LQ, Zhu XH, Yi H, Cui SY, Zhao JN, Cui ZM. miR-135b expression downregulates Ppm1e to activate AMPK signaling and protect osteoblastic cells from dexamethasone. Oncotarget. 2016; 7:7061370622. doi: 10.18632/oncotarget.12138.

21. Du H, Guo L, Fang F, Chen D, Sosunov AA, McKhann GM, Yan Y, Wang C, Zhang H, Molkentin JD, Gunn-Moore FJ, Vonsattel JP, Arancio O, et al. Cyclophilin D deficiency attenuates mitochondrial and neuronal perturbation and ameliorates learning and memory in Alzheimer's disease. Nat Med. 2008; 14:1097-1105.

22. Huang AL, Ostrowski MC, Berard D, Hager GL. Glucocorticoid regulation of the Ha-MuSV p21 gene conferred by sequences from mouse mammary tumor virus. Cell. 1981; 27:245-255.

23. Shackelford DB, Shaw RJ. The LKB1-AMPK pathway: metabolism and growth control in tumour suppression. Nat Rev Cancer. 2009; 9:563-575.

24. Luo Z, Saha AK, Xiang X, Ruderman NB.AMPK, the metabolic syndrome and cancer.Trends Pharmacol Sci. 2005; 26:69-76.

25. Faubert B, Vincent EE, Poffenberger MC, Jones RG. The AMP-activated protein kinase (AMPK) and cancer: many faces of a metabolic regulator. Cancer Lett. 2015; 356:165-170.

26. Choi YK, Park KG. Metabolic roles of AMPK and metformin in cancer cells. Mol Cells. 2013; 36:279-287.

27. Wang B, Wang XB, Chen LY, Huang L and Dong RZ. Belinostat-induced apoptosis and growth inhibition in pancreatic cancer cells involve activation of TAK1-AMPK signaling axis. Biochem Biophys Res Commun. 2013; 437:1-6.

28. Hardie DG, Ross FA, Hawley SA. AMP-Activated Protein Kinase: A Target for Drugs both Ancient and Modern. Chem Biol. 2012; 19:1222-1236.

29. Chang CH, Lee CY, Lu CC, Tsai FJ, Hsu YM, Tsao JW, Juan YN, Chiu HY, Yang JS, Wang CC. Resveratrol-induced autophagy and apoptosis in cisplatin-resistant human oral cancer CAR cells: A key role of AMPK and Akt/mTOR signaling. Int J Oncol. 2017; 50:873-882.

30. Ji C, Yang B, Yang YL, He SH, Miao DS, He L, Bi ZG. Exogenous cell-permeable C6 ceramide sensitizes multiple cancer cell lines to Doxorubicin-induced apoptosis by promoting AMPK activation and mTORC1 inhibition. Oncogene. 2010; 29:6557-6568.

31. Chen L, Xu B, Liu L, Luo Y, Yin J, Zhou H, Chen W, Shen T, Han X, Huang S. Hydrogen peroxide inhibits mTOR signaling by activation of AMPKalpha leading to apoptosis of neuronal cells. Lab Invest. 2010; 90:762-773.

32. Kimura N, Tokunaga C, Dalal S, Richardson C, Yoshino K, Hara K, Kemp BE, Witters LA, Mimura O, Yonezawa K. A possible linkage between AMP-activated protein kinase (AMPK) and mammalian target of rapamycin (mTOR) signalling pathway. Genes Cells. 2003; 8:65-79.

33. Inoki K, Zhu T, Guan KL. TSC2 mediates cellular energy response to control cell growth and survival. Cell. 2003; 115:577-590.

34. Zhang WB, Wang Z, Shu F, Jin YH, Liu HY, Wang QJ, Yang Y. Activation of AMP-activated protein kinase by temozolomide contributes to apoptosis in glioblastoma cells via 553 activation and mTORC1 inhibition. J Biol Chem. 2010; 285:40461-40471.

35. Yang L, Zheng LY, Tian Y, Zhang ZQ, Dong WL, Wang XF, Zhang XY, Cao C. C6 ceramide dramatically enhances docetaxel-induced growth inhibition and apoptosis in cultured breast cancer cells: a mechanism study. Exp Cell Res. 2015; 332:47-59.

36. Chen MB, Shen WX, Yang Y, Wu XY, Gu JH, Lu PH. Activation of AMP-activated protein kinase is involved in vincristine-induced cell apoptosis in B16 melanoma cell. J Cell Physiol. 2011; 226:1915-1925.

37. Jones RG, Plas DR, Kubek S, Buzzai M, Mu J, Xu Y, Birnbaum MJ, Thompson CB. AMP-activated protein kinase induces a p53-dependent metabolic checkpoint. Mol Cell. 2005; 18:283-293.

38. Zheng QY, Jin FS, Yao C, Zhang T, Zhang GH, Ai X. Ursolic acid-induced AMP-activated protein kinase (AMPK) activation contributes to growth inhibition and apoptosis in human bladder cancer T24 cells. Biochem Biophys Res Commun. 2012; 419:741-747.

39. Sun H, Yu T, Li J. Co-administration of perifosine with paclitaxel synergistically induces apoptosis in ovarian cancer cells: more than just AKT inhibition. Cancer Lett. 2011; 310:118-128.

40. Ji C, Yang YL, Yang Z, Tu Y, Cheng L, Chen B, Xia JP, Sun WL, Su ZL, He L, Bi ZG. Perifosine sensitizes UVBinduced apoptosis in skin cells: new implication of skin cancer prevention? Cell Signal. 2012; 24:1781-1789.

41. Kim J, Kundu M, Viollet B, Guan KL. AMPK and mTOR regulate autophagy through direct phosphorylation of Ulk1. Nat Cell Biol. 2011; 13:132-141.

42. Chen MB, Wu XY, Gu JH, Guo QT, Shen WX, Lu PH. Activation of AMP-activated protein kinase contributes to doxorubicin-induced cell death and apoptosis in cultured myocardial H9c2 cells. Cell Biochem Biophys. 2010; 60:311-322. 
43. Rocha GZ, Dias MM, Ropelle ER, Osorio-Costa F, Rossato FA, Vercesi AE, Saad MJ, Carvalheira JB. Metformin amplifies chemotherapy-induced AMPK activation and antitumoral growth. Clin Cancer Res. 2011; 17:3993-4005.

44. Zhai L, Sun N, Han Z, Jin HC, Zhang B. Liposomal shortchain C6 ceramide induces potent anti-osteosarcoma activity in vitro and in vivo. Biochem Biophys Res Commun. 2015; 468:274-280.

45. Li ZW, Cai S, Liu Y, Yang CL, Tian Y, Chen G, Cao C. Overexpression of Galphai3 in human glioma is required for AktmTOR activation and cell growth. Oncotarget. 2016 Aug 1. doi: 10.18632/oncotarget.10995. [Epub ahead of print].

46. Livak KJ, Schmittgen TD. Analysis of relative gene expression data using real-time quantitative PCR and the 2(-Delta DeltaC(T)) Method. Methods. 2001; 25:402-408.

47. Xu Z, Han Y, Liu J, Jiang F, Hu H, Wang Y, Liu Q, Gong Y, Li X. MiR-135b-5p and MiR-499a-3p Promote Cell Proliferation and Migration in Atherosclerosis by Directly Targeting MEF2C. Sci Rep. 2015; 5:12276.

48. Valeri N, Braconi C, Gasparini P, Murgia C, Lampis A, Paulus-Hock V, Hart JR, Ueno L, Grivennikov SI, Lovat F,
Paone A, Cascione L, Sumani KM, et al. MicroRNA-135b promotes cancer progression by acting as a downstream effector of oncogenic pathways in colon cancer. Cancer Cell. 2014; 25:469-483.

49. Umezu T, Tadokoro H, Azuma K, Yoshizawa S, Ohyashiki K, Ohyashiki JH. Exosomal miR-135b shed from hypoxic multiple myeloma cells enhances angiogenesis by targeting factor-inhibiting HIF-1. Blood. 2014; 124:3748-3757.

50. Zhu YR, Min H, Fang JF, Zhou F, Deng XW, Zhang YQ. Activity of the novel dual phosphatidylinositol 3-kinase/ mammalian target of rapamycin inhibitor NVP-BEZ235 against osteosarcoma.Cancer Biol Ther. 2015; 16:602-609.

51. Zhu YR, Zhou XZ, Zhu LQ, Yao C, Fang JF, Zhou F, Deng XW, Zhang YQ. The anti-cancer activity of the $\mathrm{mTORC} 1 / 2$ dual inhibitor XL388 in preclinical osteosarcoma models. Oncotarget. 2016; 7:49527-49538. doi: 10.18632/oncotarget.10389.

52. Zhu YR, Xu Y, Fang JF, Zhou F, Deng XW, Zhang YQ. Bufotalin-induced apoptosis in osteoblastoma cells is associated with endoplasmic reticulum stress activation. Biochem Biophys Res Commun. 2014; 451:112-118. 